\title{
Radiation hardness of Ce-doped sol-gel silica fibers for High Energy Physics applications
}

\author{
Francesca Cova ${ }^{1}$, Federico Moretti ${ }^{1,},{ }^{* * *}$, MaUro Fasoli $^{1}$, Norberto Chiodini ${ }^{1}$, Kristof \\ Pauwels $^{2}$, Etiennette Auffray ${ }^{3}$, Marco Toliman Lucchini ${ }^{3}$, Stefania Baccaro ${ }^{4}$, Alessia \\ Cemmi $^{4}$, Hana BÁRTOVÁ ${ }^{5}$, And AnNa Vedda ${ }^{1}$ \\ ${ }^{1}$ Department of Materials Science, University of Milano-Bicocca, Via Cozzi 55, 20125 Milano (Italy) \\ ${ }^{2}$ Department of Physics, University of Milano-Bicocca, Piazza della Scienza 3, 20125 Milano (Italy) \\ ${ }^{3}$ CERN, Route de Meyrin, 1211 Geneva 23 (Switzerland) \\ ${ }^{4}$ ENEA FSN, Casaccia, Via Anguillarese 301, 00123 Roma (Italy) \\ ${ }^{5}$ Faculty of Nuclear Sciences and Physical Engineering, Czech Technical University in Prague, Brehova 7, Prague 1 (Czech Republic) \\ ${ }^{* *}$ Now at Materials Science Division, Lawrence Berkeley National Laboratory, 1 Cyclotron Road Mailstop M/S 55R0121, Berkeley, CA 94720 (USA) \\ ${ }^{*}$ Corresponding author: fmoretti@lbl.gov
}

Compiled January 16, 2018

The results of irradiation tests on Ce-doped sol-gel silica using $\mathrm{X}$ - and $\gamma$-rays up to $\mathbf{1 0} \mathrm{kGy}$ are reported, in order to investigate the radiation hardness of this material for high energy physics applications. Sol-gel silica fibers with Ce concentrations of $0.0125 \mathrm{~mol} \%$ and $0.05 \mathrm{~mol} \%$ are characterized by means of optical absorption and attenuation length measurements before and after irradiation. The two different techniques give comparable results, evidencing the formation of a main broad radiation-induced absorption band, peaking at about $2.2 \mathrm{eV}$, related to radiation-induced color centers. The results are compared with those obtained on bulk silica. This study reveals that an improvement of the radiation hardness of Ce-doped silica fibers can be achieved by reducing Ce content inside the fiber core, paving the way for further material development. (C) 2018 Optical Society of America

OCIS codes: (060.2290) Fiber materials; (060.2300) Fiber measurements; (300.1030) Absorption; (260.3800) Luminescence.

http://dx.doi.org/10.1364/ao.XX.XXXXXX

Recent studies demonstrated that rare-earth (RE) doped glasses prepared by sol-gel route are suitable materials for the realization of scintillating optical fiber sensors, opening their application perspectives for real time dosimetry in medical systems [1], as well as for high energy physics (HEP) applications. Silica fibers could be applied as wavelength shifters for the collection and transport of light in HEP experiments [2]. Their use as the active scintillating material in a sampling electromagnetic Spaghetti Calorimeter (SPACAL) [3,4] or as the scintillating component in a dual-readout calorimeter [5, 6], coupled with undoped fibers exploiting Cherenkov light, has also been recently proposed.
An extremely good radiation hardness is a crucial property of the material for such applications: the most challenging requirements are expected in the High Luminosity Large Hadron Collider phase (HL-LHC) [7], in which the radiationinduced absorption coefficient of the scintillator material should be kept below 1-2 $\mathrm{m}^{-1}$ even after a cumulated dose of $300 \mathrm{kGy}$. In the last years, it has been proposed that scintillators based on glass matrices could be a good alternative to several crystals, for their easy preparation, shaping possibilities, and lower costs of production $[8,9]$. Moreover, glass synthesis by sol-gel technique can be performed by using high purity precursors, reducing the level of unwanted impurities that can give rise to extrinsic color centers under irradiation [10].

The degradation of the attenuation length of Ce-doped crystalline matrices after irradiation has been intensively studied: LuAG:Ce [6, 11] and YAG:Ce [12] single crystal fibers have been characterized under high levels of $\gamma$ and proton irradiation fields. On the other hand, it has never been investigated in Cedoped silica fibers.

In this work, we present a detailed study of the optical properties under irradiation and of the radiation resistance of Ce-doped silica fibers, as the results of irradiation tests using $\gamma$-rays from a ${ }^{60}$ Co source and X-rays up to an integrated dose of several kGy.

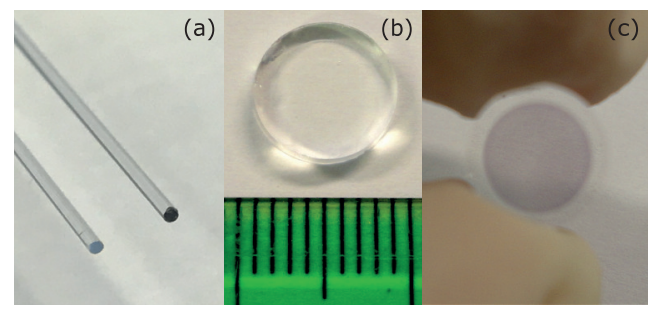

Fig. 1. Pictures of $0.05 \%$ Ce-doped optical fibers (a) before (on the left) and after $1 \mathrm{kGy}$ irradiation. Panels $\mathrm{b}$ and $\mathrm{c}$ report the parent silica glass bulk sample before and after irradiation, respectively. 


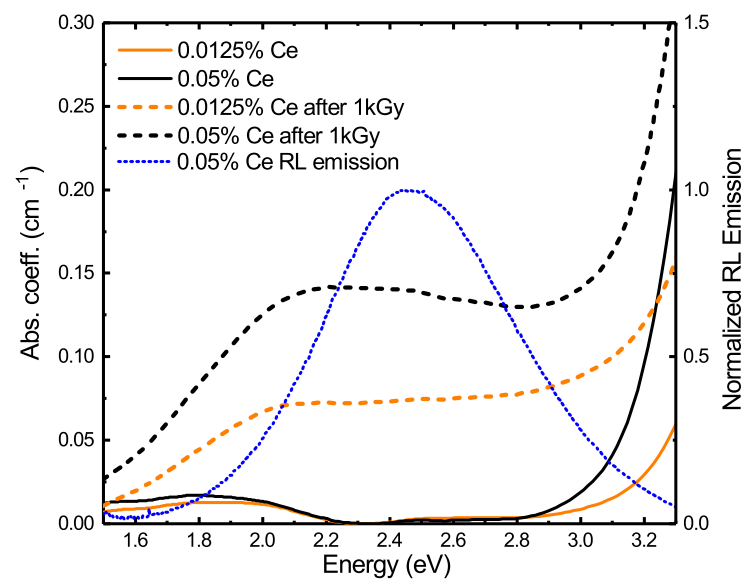

Fig. 2. Optical absorption spectra of $\mathrm{SiO}_{2}: 0.0125 \% \mathrm{Ce}$ - and $\mathrm{SiO}_{2}: 0.05 \%$ Ce-doped fibers before (solid lines) and after (dashed lines) $1 \mathrm{kGy} \gamma$ irradiation. Dotted line: RL emission spectrum of $0.05 \%$ Ce-doped silica fiber.

Ce-doped silica glasses with $\mathrm{Ce}$ concentrations of $0.0125 \mathrm{~mol} \%$ and $0.05 \mathrm{~mol} \%$ were prepared by the sol-gel method using tetramethylorthosilicate (TMOS) and Ce(III) nitrate as precursors. Alcogels were obtained after gelation and subsequently dried in a thermostatic chamber for a few weeks. The obtained xerogels were densified at $1225{ }^{\circ} \mathrm{C}$ in oxidizing $\left(\mathrm{O}_{2}\right)$ atmosphere, in order to obtain Ce-doped preforms with dimensions of $70 \mathrm{~mm}$ in length and $10 \mathrm{~mm}$ diameter. The preforms were then drawn at a temperature of about $1900 \mathrm{~K}$ into fiber by Polymicro Technologies (Phoenix, USA), using a fluorinated $\mathrm{SiO}_{2}$ cladding wrapping the Ce-doped core, in order to guarantee the light guiding by the core-cladding interface. The fibers were then left uncoated. The final core diameter is $0.60 \mathrm{~mm}$ and the final fiber diameter is $0.75 \mathrm{~mm}$. Fibers were cut into $20 \mathrm{~cm}$ pieces for measurement purposes.

In Fig. 1, pictures of a Ce-doped fiber and the parent glass bulk sample before and after irradiation are shown. After the exposure to ionizing radiation, the sample displayed coloration mostly in the Ce-doped core, where the luminescent activator is present. Therefore, we suggest that defects responsible for the formation of radiation-induced absorption bands in the visible range are likely related to the RE presence, as already observed for Pr-doped silica glasses [13].

Fiber samples were investigated by means of optical absorption (OA) spectroscopy using a Perkin Elmer Lambda 950 UV/VIS Spectrometer featuring a Transfer-Optic for 1X/2X/3X/4X/Bio (Part No. B0509546) accessory for fiber measurements. The fibers were equipped with FC connectors at both the extremities and were enclosed in a black heat-shrinkable case to eliminate possible cladding modes. The irradiation of the $\mathrm{SiO}_{2}: 0.0125 \% \mathrm{Ce}$-doped fiber was carried out at the Faculty of Nuclear Sciences and Physical Engineering (Prague, Czech Republic) with a dose rate of approximately $40 \mathrm{~Gy} / \mathrm{h}$, and the irradiation of the $\mathrm{SiO}_{2}: 0.05 \%$ Ce-doped fiber was performed at the IONISOS irradiation facility (Dagneux, Lyon, France) with a dose rate of $1 \mathrm{kGy} / \mathrm{h}$; in both cases, a ${ }^{60}$ Co source was used and the cumulated dose was $1 \mathrm{kGy}$.

In Fig. 2 the OA spectra of $\mathrm{SiO}_{2}: 0.0125 \% \mathrm{Ce}-$ and $\mathrm{SiO}_{2}: 0.05 \%$ Ce-doped fibers before and after $1 \mathrm{kGy} \gamma$ irradiation are reported. The typical radio-luminescence (RL) spectrum of these fibers is reported as well for reference; the absolute intensities were found to be proportional to the Ce content. The measurements are limited to $3.3 \mathrm{eV}$ because the interesting region for $\mathrm{Ce}^{3+}$ luminescence is in the visible range. Moreover, at higher energies, the measurements have poor reliability because of absorbance saturation and distortions related to luminescence contribution. All the spectra are characterized by an evident absorption just above $3.1 \mathrm{eV}$ which is likely related to charge transfer of $\mathrm{Ce}^{4+}$ as well as $\mathrm{Ce}^{3+} 4 \mathrm{f}-5 \mathrm{~d}$ transition [14]. Moreover, a broad and weak absorption structure is visible in the non-irradiated samples below $2.2 \mathrm{eV}$. Preliminary results obtained on Pr-doped sol-gel silica fibers showed the presence of similar absorption contributions, even more evident than in the case of the here reported Ce-doped fibers. Therefore, this broad band, here put in evidence by the high aspect ratio of fiber samples, could be more likely related to intrinsic defects, present independently from the doping [10, 15].

The irradiated samples show an evident increase of the absorption coefficient in the $1.5-3.0 \mathrm{eV}$ energy range, due to the formation of a very broad radiation-induced absorption structure: the maximum absorption coefficient evaluated at $2.2 \mathrm{eV}$ is $0.07 \mathrm{~cm}^{-1}$ and $0.14 \mathrm{~cm}^{-1}$ for the $\mathrm{SiO}_{2}: 0.0125 \%$ $\mathrm{Ce}-$ and $\mathrm{SiO}_{2}: 0.05 \% \mathrm{Ce}$-doped fibers respectively. This new absorption contribution is superimposed to the typical RL spectrum of $\mathrm{Ce}^{3+}$, leading to a reduction of the transmitted scintillation light. In this respect, we remark that a lower energy emission in the near IR would be much less affected by radiation damage of the fibers.

The radiation-induced absorption coefficient is clearly lower for the less concentrated Ce-doped fiber sample: this points to a dominant role of the reduction of the Ce content in the improvement of the radiation hardness. It is also the cause of a shift towards higher energies of the absorption edge.

These results are supported by measurements of the attenuation length of photo-luminescence (PL) emission, chosen as the main practical parameter to characterize the fibers, due to their small cross-section and high aspect ratio. The measurements were performed with a homemade setup, by illuminating the fiber with a $370 \mathrm{~nm}$ pulsed LED moving at constant speed $(2.6 \mathrm{~cm} / \mathrm{s})$ along its longitudinal axis, as shown in Fig. 3, and monitoring the luminescence output through dry coupling both fiber ends to two Hamamatsu H6610 photomultiplier tubes (PMT) working at $2.3 \mathrm{kV}$. Long-pass filters (ThorLabs FGL420) were used to avoid parasitic excitation light. The LED was coupled through an optical fiber to a collimator, providing a beam size of about 2-3 mm. The LED pulse duration was $150 \mathrm{~ns}$ and the frequency $10 \mathrm{kHz}$. The double-side readout of the light allows to correct the measurement in case of small variations of the LED intensity and to compensate for fluctuations along the fiber, improving the reliability of the attenuation measurement and simplifying the readout procedure, as discussed in [6].

The results are shown in Fig. 4. The attenuation length curves follow a single exponential decay according to Eq. 1, where $S_{\text {right }}$ and $S_{\text {left }}$ are the signal intensities recorded by the two photodetectors, $1_{\text {att }}$ is the attenuation length of the fiber, defined as the distance at which the incident beam is reduced of a factor

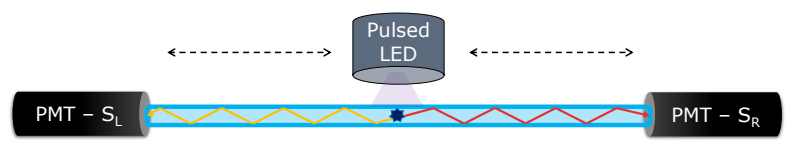

Fig. 3. Setup for the attenuation length measurement based on double-side readout: the pulsed LED light source at about $1 \mathrm{~cm}$ distance is moved along the longitudinal axis of the fiber. 

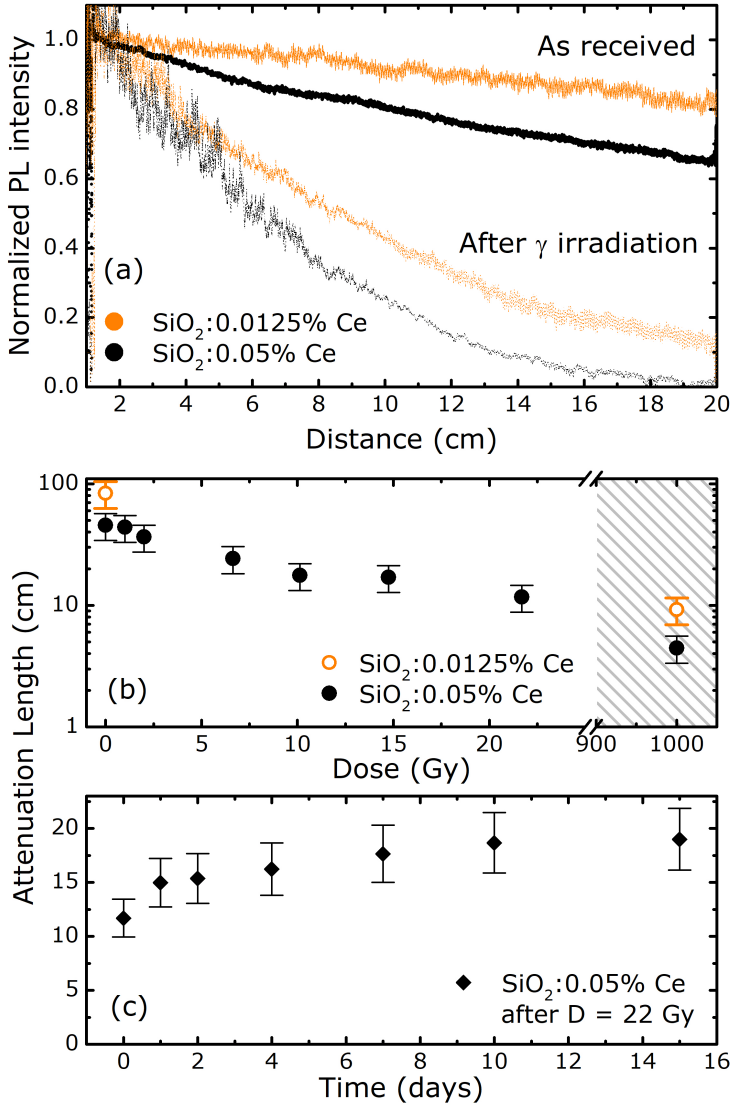

Fig. 4. (a) Attenuation length curves for $\mathrm{SiO}_{2}: 0.0125 \% \mathrm{Ce}-$ and $\mathrm{SiO}_{2}: 0.05 \%$ Ce-doped fibers before (solid lines) and after (dotted lines) $1 \mathrm{kGy} \gamma$ irradiation.

Bottom panels: attenuation length values as a function of dose (b) and of RT recovery time after 22 Gy irradiation (c).

$1 / \mathrm{e}$, and $x$ is the position along the fiber.

$$
\frac{S_{\text {right }}}{S_{\text {left }}}=A_{0} \exp \left(\frac{-2 x}{l_{\text {att }}}\right)
$$

In practice, the reciprocal of the attenuation length has a physical meaning similar to that of the absorption coefficient for spectrally unresolved measurements.

Panel (a) of Fig. 4 displays the attenuation length curves for $\mathrm{SiO}_{2}: 0.0125 \% \mathrm{Ce}$ - and $\mathrm{SiO}_{2}: 0.05 \%$ Ce-doped fibers: the reduction of $\mathrm{Ce}$ concentration improves the attenuation length of the glass fibers both before and after $1 \mathrm{kGy} \gamma$ irradiation, performed at the IONISOS irradiation facility in both cases. In fact, the attenuation length was estimated to be around $80 \mathrm{~cm}$ and $45 \mathrm{~cm}$ before irradiation and around $10 \mathrm{~cm}$ and $5 \mathrm{~cm}$ after irradiation for the $\mathrm{SiO}_{2}: 0.0125 \% \mathrm{Ce}$ - and $\mathrm{SiO}_{2}: 0.05 \%$ Ce-doped fibers respectively (panel (b) of Fig. 4). Following several repetitions of the experiment, a statistical uncertainty of $25 \%$ has been associated to the attenuation length values.

We underline that attenuation length results are in good agreement with OA measurements, confirming an improvement of the radiation hardness of silica fibers with the reduction of Ce concentration. In fact, the attenuation length values after irradiation $(10$ and $5 \mathrm{~cm}$ ) are rather consistent with the absorption coefficient values in the maximum of the visible region $(0.07$ and $\left.0.14 \mathrm{~cm}^{-1}\right)$. The two methods allow a direct comparison of the response of the investigated materials in fiber shape.
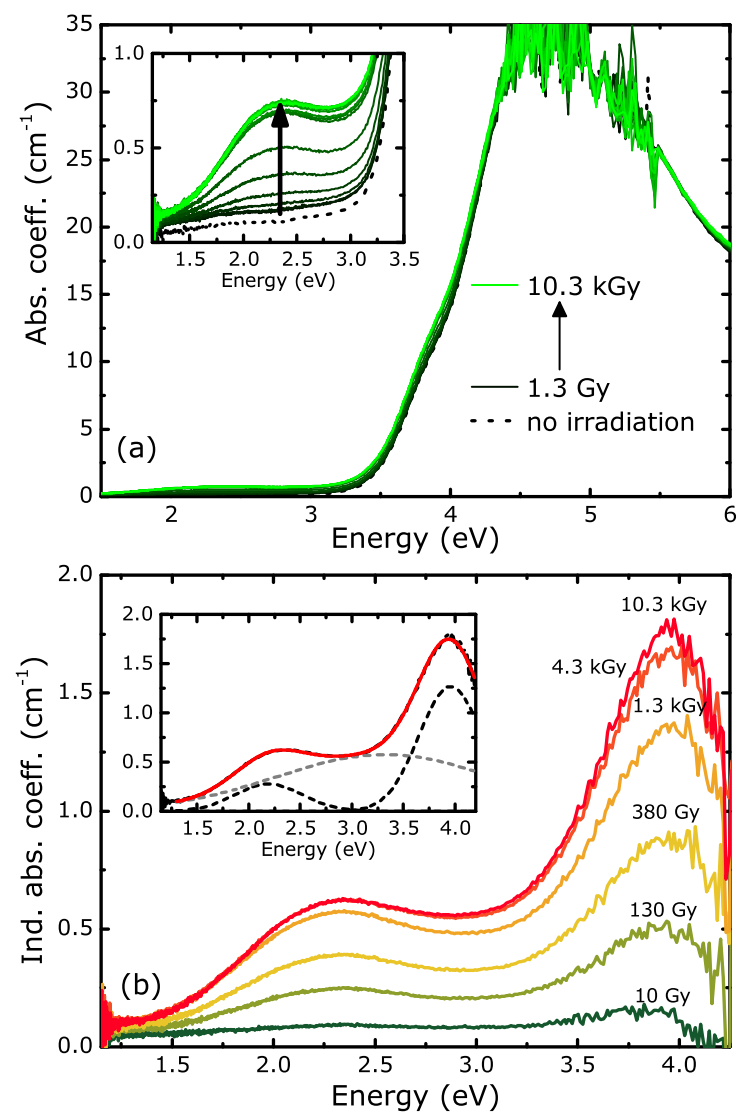

Fig. 5. (a) Optical absorption spectra of $\mathrm{SiO}_{2}: 0.05 \%$ Ce glass sample; in the inset, an enlargement of the $1.0-3.5 \mathrm{eV}$ region is displayed. The arrow indicates increasing dose. (b) Radiationinduced absorption spectra of $\mathrm{SiO}_{2}: 0.05 \%$ Ce glass sample; in the inset, numerical fit (red solid line) of the radiation-induced absorption spectrum after $10.3 \mathrm{kGy}$ carried out as a sum of three Gaussian components (black and gray dashed lines).

Attenuation length measurements permit to detect the light emitted directly by luminescent centers, reproducing conditions more similar to those found during the effective operation of HEP detectors. However, the evaluation of the attenuation does not consider variations in the emitted light spectral shape, but only a uniform reduction of the luminescence intensity. The two techniques can thus be regarded as complementary in the estimation of the absorption features of the fibers under investigation.

The attenuation length trend as a function of dose is reported in panel (b) of Fig. 4: in this case, a $\gamma$ irradiation up to 22 Gy was performed at a CERN irradiation facility with a ${ }^{60}$ Co source at a low dose rate of about $1 \mathrm{~Gy} /$ day. The shaded region of the figure shows the attenuation length values after $1 \mathrm{kGy}$ irradiation performed at the IONISOS irradiation facility.

Panel (c) of Fig. 4 displays the attenuation length as a function of room temperature (RT) recovery time after $22 \mathrm{~Gy}$ irradiation: a recovery of about $22 \%$ followed over 15 days can be observed.

For a deeper understanding of the optical absorption response, the OA spectra as a function of irradiation dose of a sample cut from the residual of fiber drawing process (denoted in the following as melted sample), with dimensions $2.5 \mathrm{~mm}$ in thickness and $8.5 \mathrm{~mm}$ diameter, were also studied using a Varian Cary 50 Spectrophotometer, and the results are reported 


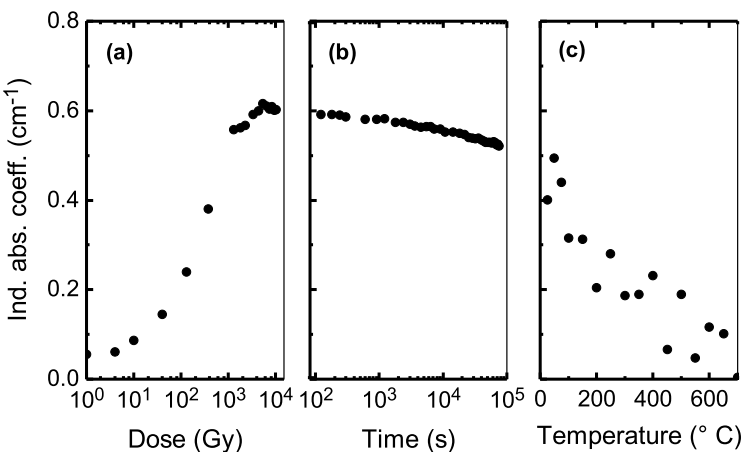

Fig. 6. Radiation-induced absorption coefficient at $2.2 \mathrm{eV}$ of $\mathrm{SiO}_{2}: 0.05 \%$ Ce-doped melted sample as a function of (a) dose, (b) RT recovery time and (c) annealing temperature. The ordinate scales are the same in the three panels.

in Fig. $5(a, b)$. For practical aspects, X-ray irradiation was employed for these measurements and it was performed by a Machlett OEG50 X-ray tube with tungsten anode, operated at $32 \mathrm{kV}$. The doses were evaluated by a PTW Duplex calibrated ionization chamber. Similar results have been obtained by few irradiations with ${ }^{60} \mathrm{Co} \gamma$ source at IONISOS and ENEA Calliope (Casaccia R. C., Rome, Italy) irradiation facilities [16].

In Fig. 5, both absorption spectra (a) and radiation-induced ones (b) are shown. The latter have been calculated as the differences between the spectra after irradiation and the spectrum before irradiation. A monotonic increase of absorption as a function of dose is observed, suggesting the occurrence of a saturation of the absorption at around 5 kGy, also evident in panel (a) of Fig. 6. The inset of panel (b) of Fig. 5 shows the numerical fit carried out on the OA spectrum obtained after $10.3 \mathrm{kGy}$ irradiation. It can be analyzed as a sum of three Gaussian components, peaking at $2.2 \mathrm{eV}, 3.3 \mathrm{eV}$, and $3.9 \mathrm{eV}$ with full width at half maximum (FWHM) $0.8 \mathrm{eV}, 2.5 \mathrm{eV}$, and $0.7 \mathrm{eV}$ respectively; the very broad width of the bands points towards the presence of contributions from defect distributions. The two lower energy bands overlap with the emission spectrum of Ce-doped silica (dotted line of Fig. 2), and can thus cause re-absorption of the emitted light.

The OA spectra of the Ce-doped melted sample and fibers show a similar absorption shape, suggesting that the drawing process does not modify the nature of the centers responsible for the radiation-induced absorptions. We put in evidence that the maximum absorption coefficient in the visible region is higher by about a factor 4 in the $0.05 \%$ Ce-doped melted sample with respect to the corresponding fiber. On the other hand, it is a factor 7 lower than for Pr-doped sol-gel silica bulk samples [13], making the Ce-doped silica a more radiation hard material with respect to the Pr-doped one.

The evolutions of the absorption coefficient at $2.2 \mathrm{eV}$ as a function of dose, RT recovery time, and annealing temperature are reported in Fig. 6. As already noticed, the induced absorption increases monotonically with increasing dose, reaching a saturation level after about $5 \mathrm{kGy}$. The behavior of the OA spectra as a function of recovery time was monitored for a total time of about 20 hours. The absorption decrease turned out to be about $10 \%$, as shown in panel (b) of Fig. 6 . This recovery rate is comparable to that of fibers, estimated to be around $10 \%$ after 1 day monitoring. The observation of RT recovery indicates that a dynamical equilibrium between color centers filling and RT release is established which actually could depend upon the dose rate. Indeed, the spontaneous RT recovery is an important feature because it reduces the radiation damage effect without requiring any treatment on the sample. In addition, thermally activated recovery was also investigated by annealing cycles up to $700{ }^{\circ} \mathrm{C}$ performed by heating up the Ce-doped melted sample in steps of $50{ }^{\circ} \mathrm{C}$ by holding it in a preheated oven for $15 \mathrm{~min}$ at each temperature step. A complete recovery of the radiation damage was observed at $700{ }^{\circ} \mathrm{C}$.

In conclusion, our study suggests that the radiation resistance of Ce-doped silica is enhanced by the fiber drawing process, probably due to a silica network rearrangement and a consequent reduction of defect concentrations occurring during this high temperature process. Moreover, both trough a spectroscopic and a practical approach, it is shown that also a reduction of $\mathrm{Ce}$ content inside the silica fiber core leads to an improvement of the radiation hardness, suggesting that radiation-induced defects are related to the presence of the dopant.

These results can guide the future engineering of scintillating silica fibers, that will take into account the possibility to reach a satisfactory optimization of both emission intensity and radiation hardness.

Funding This work has been supported by the $\mathrm{H} 2020$ projects AIDA-2020 (GA no. 654168), INTELUM (GA no. 644260), ASCIMAT (GA no. 690599), and SPARK (GA no. 16290).

\section{REFERENCES}

1. I. Veronese, N. Chiodini, S. Cialdi, E. D'Ippolito, M. Fasoli, S. Gallo, S. La Torre, E. Mones, A. Vedda, and G. Loi, Phys. Med. Biol. 62, 4218 (2017).

2. R. Becker, G. Dissertori, A. Gendotti, Q. Huang, D. Luckey, W. Lustermann, S. Lutterer, F. Nessi-Tedaldi, F. Pandolfi, F. Pauss, M. Peruzzi, M. Quittnat, and R. Wallny, J. Phys.: Conf. Ser. 587, 012039 (2015).

3. A. Benaglia, M. Lucchini, K. Pauwels, C. Tully, T. Medvedeva, A. Heering, C. Dujardin, V. Kononets, K. Lebbou, N. Aubry, S. Faraj, G. Ferro, P. Lecoq, and E. Auffray, J. Instrum. 11, P05004 (2016).

4. M. Lucchini, T. Medvedeva, K. Pauwels, C. Tully, A. Heering, C. Dujardin, K. Lebbou, P. Lecoq, and E. Auffray, J. Instrum. 8, P10017 (2013).

5. M. Cascella, S. Franchino, and S. Lee, "Fiber and crystals dual readout calorimeters," in "The Future of High Energy Physics - Some Aspects," (World Scientific Publishing Co. Pte. Ltd., 2017), p. 277.

6. K. Pauwels, C. Dujardin, S. Gundacker, K. Lebbou, P. Lecoq, M. Lucchini, F. Moretti, A. G. Petrosyan, X. Xu, and E. Auffray, J. Instrum. 8, P09019 (2013).

7. D. Contardo, M. Klute, J. Mans, L. Silvestris, and J. Butler, "Technical Proposal for the Phase-II Upgrade of the CMS detector," Tech. Rep. CERN-LHCC-2015-010. LHCC-P-008. CMS-TDR-15-02, CERN, Geneva (2015).

8. M. J. F. Empizo, M. Cadatal-Raduban, T. Murata, Y. Minami, K. Kawano, K. Yamanoi, T. Shimizu, N. Sarukura, M. Guzik, Y. Guyot, and G. Boulon, J. Lumin., http://dx.doi.org/10.1016/j.jlumin.2017.06.029 (2017).

9. T. Yanagida, J. Ueda, H. Masai, Y. Fujimoto, and S. Tanabe, J. Non-Cryst. Solids 431, 140 (2016).

10. A. Vedda, N. Chiodini, D. Di Martino, M. Fasoli, F. Morazzoni, F. Moretti, R. Scotti, G. Spinolo, A. Baraldi, R. Capelletti, M. Mazzera, and M. Nikl, Chem. Mater. 18, 6178 (2006).

11. V. Kononets, E. Auffray, C. Dujardin, S. Gridin, F. Moretti, G. Patton, K. Pauwels, O. Sidletskiy, X. Xu, and K. Lebbou, J. Cryst. Growth 435, 31 (2016).

12. M. Lucchini, K. Pauwels, K. Blazek, S. Ochesanu, and E. Auffray, IEEE Trans. Nucl. Sci. 63, 586 (2016).

13. F. Cova, M. Fasoli, F. Moretti, N. Chiodini, K. Pauwels, E. Auffray, M. Lucchini, E. Bourret, I. Veronese, E. d'Ippolito, and A. Vedda, J. Lumin. 192, 661 (2017).

14. M. Fasoli, A. Vedda, A. Lauria, F. Moretti, E. Rizzelli, N. Chiodini, F. Meinardi, and M. Nikl, J. Non-Cryst. Solids 355, 1140 (2009).

15. L. Skuja, J. Non-Cryst. Solids 239, 16 (1998).

16. S. Baccaro and A. Cemmi, J. Phys.: Conf. Ser. 763, 012001 (2016). 


\section{FULL REFERENCES}

1. I. Veronese, N. Chiodini, S. Cialdi, E. D'Ippolito, M. Fasoli, S. Gallo, S. La Torre, E. Mones, A. Vedda, and G. Loi, "Real-time dosimetry with Yb-doped silica optical fibres," Phys. Med. Biol. 62, 4218 (2017).

2. R. Becker, G. Dissertori, A. Gendotti, Q. Huang, D. Luckey, W. Lustermann, S. Lutterer, F. Nessi-Tedaldi, F. Pandolfi, F. Pauss, M. Peruzzi, M. Quittnat, and R. Wallny, "Proof-of-principle of a new geometry for sampling calorimetry using inorganic scintillator plates," J. Phys.: Conf. Ser. 587, 012039 (2015).

3. A. Benaglia, M. Lucchini, K. Pauwels, C. Tully, T. Medvedeva, A. Heering, C. Dujardin, V. Kononets, K. Lebbou, N. Aubry, S. Faraj, G. Ferro, P. Lecoq, and E. Auffray, "Test beam results of a high granularity LuAG fibre calorimeter prototype," J. Instrum. 11, P05004 (2016).

4. M. Lucchini, T. Medvedeva, K. Pauwels, C. Tully, A. Heering, C. Dujardin, K. Lebbou, P. Lecoq, and E. Auffray, "Test beam results with LuAG fibers for next-generation calorimeters," J. Instrum. 8, P10017 (2013).

5. M. Cascella, S. Franchino, and S. Lee, "Fiber and crystals dual readout calorimeters," in "The Future of High Energy Physics - Some Aspects," (World Scientific Publishing Co. Pte. Ltd., 2017), p. 277.

6. K. Pauwels, C. Dujardin, S. Gundacker, K. Lebbou, P. Lecoq, M. Lucchini, F. Moretti, A. G. Petrosyan, X. Xu, and E. Auffray, "Single crystalline LuAG fibers for homogeneous dual-readout calorimeters," $J$. Instrum. 8, P09019 (2013).

7. D. Contardo, M. Klute, J. Mans, L. Silvestris, and J. Butler, "Technical Proposal for the Phase-II Upgrade of the CMS detector," Tech. Rep. CERN-LHCC-2015-010. LHCC-P-008. CMS-TDR-15-02, CERN, Geneva (2015).

8. M. J. F. Empizo, M. Cadatal-Raduban, T. Murata, Y. Minami, K. Kawano, K. Yamanoi, T. Shimizu, N. Sarukura, M. Guzik, Y. Guyot, and G. Boulon, "Spectroscopic properties of $\mathrm{Pr}^{3+}$-doped 20Al $\left(\mathrm{PO}_{3}\right)_{3}-$ $80 \mathrm{LiF}$ glasses as potential scintillators for neutron detection," J. Lumin., http://dx.doi.org/10.1016/j.jlumin.2017.06.029 (2017).

9. T. Yanagida, J. Ueda, H. Masai, Y. Fujimoto, and S. Tanabe, "Optical and scintillation properties of Ce-doped $34 \mathrm{Li}_{2} \mathrm{O}-5 \mathrm{MgO}-10 \mathrm{Al}_{2} \mathrm{O}_{3}-51 \mathrm{SiO}_{2}$ glass," J. Non-Cryst. Solids 431, 140 (2016).

10. A. Vedda, N. Chiodini, D. Di Martino, M. Fasoli, F. Morazzoni, F. Moretti, R. Scotti, G. Spinolo, A. Baraldi, R. Capelletti, M. Mazzera, and M. Nikl, "Insights into microstructural features governing $\mathrm{Ce}^{3+}$ luminescence efficiency in sol-gel silica glasses," Chem. Mater. 18, 6178 (2006).

11. V. Kononets, E. Auffray, C. Dujardin, S. Gridin, F. Moretti, G. Patton, K. Pauwels, O. Sidletskiy, X. Xu, and K. Lebbou, "Growth of long undoped and Ce-doped LuAG single crystal fibers for dual readout calorimetry," J. Cryst. Growth 435, 31 (2016).

12. M. Lucchini, K. Pauwels, K. Blazek, S. Ochesanu, and E. Auffray, "Radiation Tolerance of LuAG:Ce and YAG:Ce Crystals under High Levels of Gamma-and Proton-Irradiation," IEEE Trans. Nucl. Sci. 63, 586 (2016).

13. F. Cova, M. Fasoli, F. Moretti, N. Chiodini, K. Pauwels, E. Auffray, M. Lucchini, E. Bourret, I. Veronese, E. d'Ippolito, and A. Vedda, "Optical properties and radiation hardness of $\mathrm{Pr}$-doped sol-gel silica: Influence of fiber drawing process," J. Lumin. 192, 661 (2017).

14. M. Fasoli, A. Vedda, A. Lauria, F. Moretti, E. Rizzelli, N. Chiodini, F. Meinardi, and M. Nikl, "Effect of reducing sintering atmosphere on Ce-doped sol-gel silica glasses," J. Non-Cryst. Solids 355, 1140 (2009).

15. L. Skuja, "Optically active oxygen-deficiency-related centers in amorphous silicon dioxide," J. Non-Cryst. Solids 239, 16 (1998).

16. S. Baccaro and A. Cemmi, "Optical characterization of ion-doped crystalline and glassy matrices operating under hostile environmental conditions," J. Phys.: Conf. Ser. 763, 012001 (2016). 